\title{
Compressed SUSY searches with Recursive Jigsaw Reconstruction
}

\author{
Paul Jackson* \\ University of Adelaide \\ E-mail: p.jackson@adelaide.edu.au
}

\begin{abstract}
The Recursive Jigsaw Reconstruction technique provides a powerful way to search for new physics in scenarios with multiple missing particles in the final state. By defining a specific input decay tree we demonstrate a new approach to search for supersymmetry in scenarios where the mass difference between the parent super-particles and the lightest supersymmetric daughter particles is small. Such scenarios are colloquially referred to as "compressed". The imposition of this decay tree provides a clear way to define which objects are associated with an initial-state radiation system, and those which are candidate decay products of the supersymmetric system of particles. From this choice a set of variables emerge, providing a method to distinguish compressed signals from the pernicious standard model backgrounds. We introduce this new approach and demonstrate it's power through application to searches in several final states. We further touch on the applicability of the Recursive Jigsaw method in general to new physics searches where conventional kinematic discriminators break down.
\end{abstract}

The European Physical Society Conference on High Energy Physics

5-12 July

Venice, Italy

\footnotetext{
* Speaker.
} 


\section{Introduction}

The general purpose LHC discovery experiments, ATLAS and CMS, were built with two goals in mind: the discovery and measurement of the Higgs boson and to search for evidence of beyond standard model (BSM) physics at a new scale. The first of these was achieved during Run 1 and the second is now the key challenge of the LHC physics program. Such BSM physics processes may be just out of reach of the available dataset, or may be manifested in difficult-to-measure channels. In the spirit of covering every corner of phase space, it behooves us to consider all possibilities in the techniques we employ to search for BSM physics, as perhaps further improvements can be made. With this in mind we introduce the Recursive Jigsaw Reconstruction (RJR) technique [1] which can be used to investigate BSM theories characterized by a discrete $\mathbb{Z}_{2}$ symmetry in collider experiments. The RJR method approximates recursively, event-by-event, the relevant frames of reference and introduces a new basis of kinematic and angular Jigsaw variables. Final states from R-parity conserving supersymmetry (SUSY) models are characterised by an even number of lightest supersymmetric particles (LSP), likely two, undetected by the detector and contributing to the missing transverse momentum, $\vec{\not}_{T}$. By applying the RJR technique, through a series of Jigsaw Rules we can contextualize the $\overrightarrow{\vec{H}}_{T}$, partitioning it between two decay hemispheres and extract a basis of complementary variables. These variables replace conventional, detector derived, laboratory frame variables with a more physics-driven basis that can be used to characterize events and provide, where possible, uncorrelated observables to isolate signal-like events from backgroundlike events. In compressed scenarios, the same principles can be applied to a different view of the events yielding a more relevant basis of observables.

\section{Recursive Jigsaw Reconstruction}
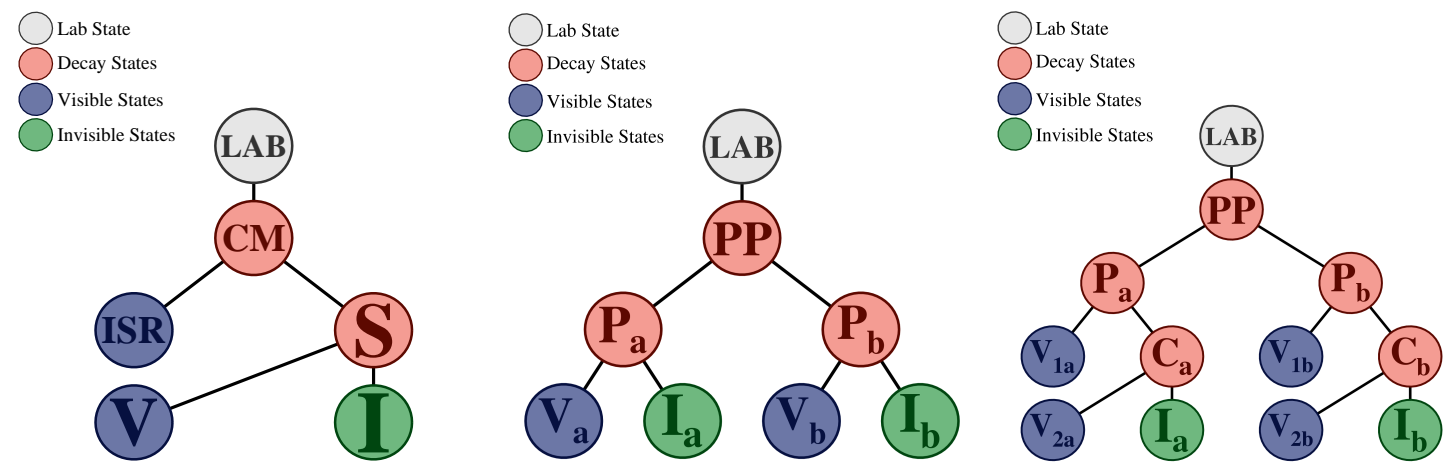

Figure 1: Decay tree diagrams for application to compressed spectra (left), pair produced parent particles " $\mathrm{P}$ " decaying to visible and invisible final states (center) and pair produced particles decaying through intermediate children systems " $\mathrm{C}$ " to sets of visible and invisible final state particles (right).

RJR is a new approach to reconstructing open final states. The strategy is to transform observable momenta iteratively reference-frame to reference-frame, traveling through each of the reference frames relevant to the topology. At each step we extremize only the relevant degrees of freedom related to that transformation. We repeat the procedure recursively according to particular rules defined for each topology (the topology relevant to each reference frame). In Figure 1 
we show three decay tree diagrams for (left) compressed searches, where the mass between the parent particle and lightest daughter particle is small, and for pair-produced systems decaying either directly (center), or through an intermediate child (right), to pairs of invisible particles. The application of the RJR algorithm and all of the "Jigsaw rules" are readily configurable through RestFrames [2] for any chosen decay tree view of an event under investigation.

\section{Application to compressed spectra}

In [3] a set of observables are introduced that provide increased sensitivity to studying compressed scenarios at collider experiments. They are designed for the analysis of events where one or more strong initial state radiation (ISR) jets are present, causing the system of initially produced sparticles to recoil in the opposite direction. In the limit where the LSPs receive no momentum from their parents' decays, the $\vec{H}_{T}$ results solely from the recoil against ISR, and the following approximation holds:

$$
\vec{B}_{T} \sim-\vec{p}_{T}^{I S R} \times \frac{m_{\tilde{\chi}}}{m_{\tilde{P}}},
$$

where $\vec{p}_{T}$ ISR is the total ISR system transverse momentum.

In the decay tree on the left in Figure 1, we can assigned each reconstructed object hypothesized to come from the decay of sparticles in the event to the "V" system, while those identified as initial state radiation are associated with "ISR". With the missing momentum reconstructed in each event interpreted as the system "I", the total sparticle system ("S") and center-of-mass system of the whole reaction ("CM") are defined as the sum of their constituents. With the four-vectors of each element of the decay tree specified, an estimator of the quantity $\left|\overrightarrow{\mathscr{E}}_{T}\right| /\left|\vec{p}_{T}^{\mathrm{ISR}}\right|, R_{\mathrm{ISR}}$, is calculated as:

$$
R_{\mathrm{ISR}} \equiv \frac{\left|\vec{p}_{\mathbf{I}, T}^{\mathbf{C M}} \cdot \hat{p}_{\mathbf{I S R}, T}^{\mathbf{C M}}\right|}{\left|\vec{p}_{\mathbf{I S R}, T}^{\mathbf{C M}}\right|},
$$

where subscripts indicate the system and superscripts the reference frame the momentum is evaluated in. Using these key variables, and others derived from the kinematic basis described in [3] it was demonstrated that gluinos(squarks) up to mass of $1400(900) \mathrm{GeV}$, with a mass difference between parents and LSP of up to $200 \mathrm{GeV}$ can be excluded with $100 \mathrm{fb}^{-1}$ of data.

In this conference I presented the first ideas of utilising this same approach to compressed physics in searches for electroweak supersymmetry. Originally included in [3] we see from Figure 2 that there is powerful discrimination with the RJR approach to more compressed supersymmetric scenarios. Given the possibility of exposing such searches to the power of the complete RJR tree, as shown in the centre of Fig 1, we propose combining these two search techniques to glean improved search potential in non-overlapping regions of phase-space.

\section{HEP event analysis in the presence of kinematic and combinatoric ambiguities}

In [1] we introduced a technique that can be applied to resolve any number of kinematic or combinatoric ambiguities in any production or decay topology of interest. This technique has been shown to provide a prescription to define physically motivated variables to make measurement of standard model processes and search for any new physics that may lie beyond the scope of 

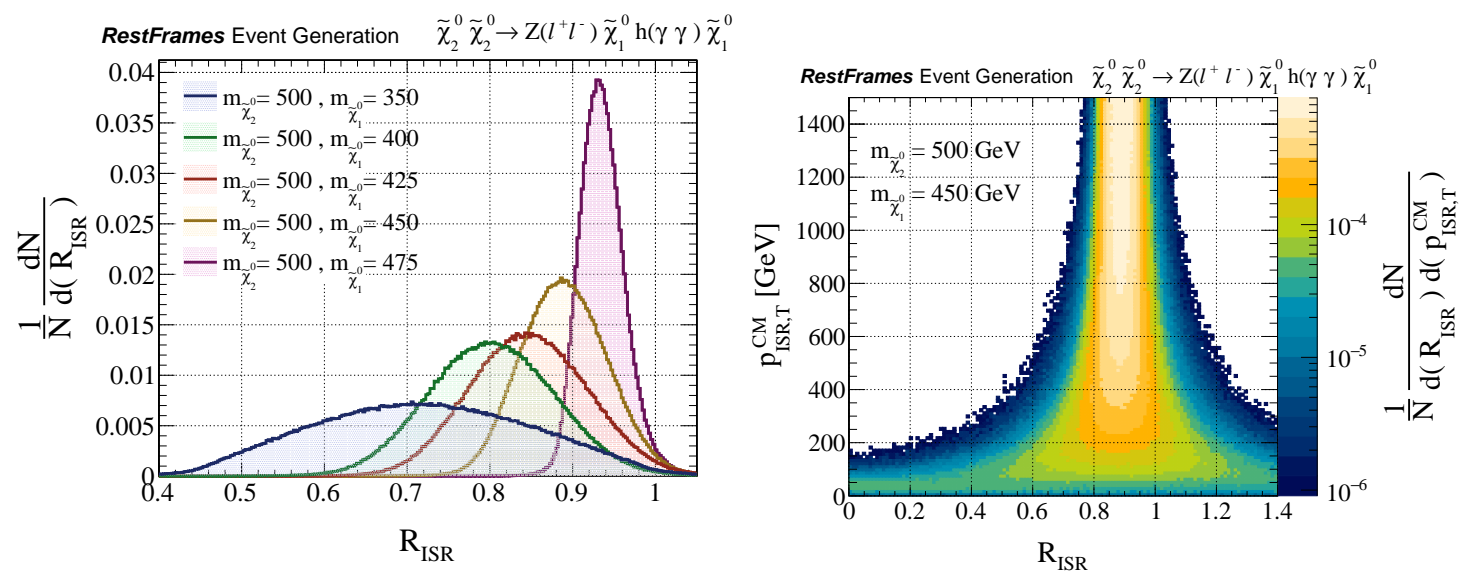

Figure 2: Distribution of the $R_{\mathrm{ISR}}$ for compressed electroweak SUSY signal (left) for various mass splittings demonstrating the efficacy of this variable in providing sensitivity to signal. A chosen signal point plotted in two-dimensions we see the strength of this variable in concert with an increased selection on the $\left|\vec{p}_{\text {ISR }, T}^{\text {CM }}\right|$.

current theory. Given the customizable nature of the approach one can easily build additional complexity into an analysis by recursively adding more information to a decay tree, while providing choices by which to break any combinatoric and kinematic ambiguities that may arise. This is demonstrated in Figure 3 for the case of the standard model Higgs decaying to $W W$, with each $W$ boson subsequently decaying leptonically. We see that we are able to reconstruct the Higgs boson mass with approximately $20 \%$ resolution. Adding a further layer of decay we can consider a heavy-Higgs decaying to pairs of standard model Higgs' with four leptonic $W$ boson decays. The parent resonance mass and intermediate masses can be measured with good accuracy but not only this, as demonstrated in Figure 33 of [1], the decay angles of the parent and intermediate particles can be reconstructed with reasonable accuracy.
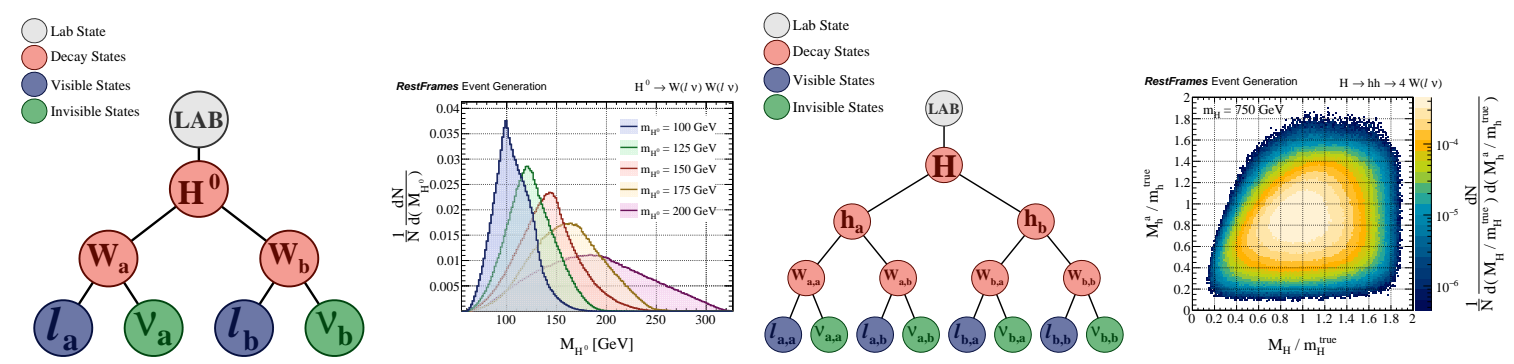

Figure 3: Decay tree diagram for standard model Higgs boson production (left) with the reconstructed Higgs mass (center left). Extending this decay tree further we build a case for a new resonance decaying to pairs of Higgs bosons (center right) and demonstrate that the particle masses can be reconstructed with similar resolution in this case (left). All Figures reproduced from [1].

Through studies of simulated samples performed in [6] much of the physics outlined in [1] has been shown to provide excellent sensitivity to improve a wide range of the physics program at a high energy collider such as the LHC. 


\section{Summary}

RJR [1] provides a way to decompose events into a complete, well-defined and largely uncorrelated basis of variables. The technique aims to remove redundancy and, as much as possible, "diagonalize" the available information to match observables with the particle masses and decay angles we hope to discover/measure. The technique has already been applied successfully to searches at the LHC for compressed SUSY in top squark decays [4] and jets $+\vec{B}_{T}$ [5]. RJR improves upon conventional analysis methods, while being complementary to them, and provides an exciting new way to search for evidence of BSM physics. Further studies of the RJR technique [6] have demonstrated improved sensitivity in searches for third generation SUSY, both in direct production and in the decay of gluinos, in the former case huge gains can be made in the compressed cases. Furthermore, $\chi_{2}^{0} \chi_{1}^{ \pm}$pair-production, decaying via intermediate $W$ and $Z$ is an interesting topology where there is considerable promise of sensitivity [6] in regions of parameter space that remain as yet unexplored.

\section{References}

[1] P. Jackson, C. Rogan, Recursive Jigsaw Reconstruction: HEP event analysis in the presence of kinematic and combinatoric ambiguities, arXiv:1705.10733.

[2] C. Rogan, RestFrames, http://RestFrames.com

[3] P. Jackson, C. Rogan and M. Santoni, Sparticles in motion: Analyzing compressed SUSY scenarios with a new method of event reconstruction, Phys. Rev. D95(3):035031, 2017 [arXiv:1607.08307].

[4] ATLAS Collaboration, Search for a scalar partner of the top quark in the jets plus missing transverse momentum final state at $\sqrt{s}=13 \mathrm{TeV}$ with the ATLAS detector, arXiv:1709.04183, submitted to JHEP.

[5] ATLAS Collaboration, Search for squarks and gluinos in final states with jets and missing transverse momentum using $36.1 \mathrm{fb}^{-1}$ of $\sqrt{\mathrm{s}}=13 \mathrm{TeV}$ pp collision data with the ATLAS detector, ATLAS-CONF-2017-022.

[6] Marco Santoni, Probing SUSY with Recursive Jigsaw Reconstruction, PhD Thesis, University of Adelaide (2017). 